\title{
Reproductive failure in mice lacking inter-alpha-trypsin inhibitor (ITI) - ITI target genes in mouse ovary identified by microarray analysis
}

\author{
Mika Suzuki, Hiroshi Kobayashi, Yoshiko Tanaka, \\ Naohiro Kanayama and Toshihiko Terao \\ Department of Obstetrics and Gynecology, Hamamatsu University School of Medicine, Handayama 1-20-1, Hamamatsu, Shizuoka, 431-3192, Japan \\ (Requests for offprints should be addressed to Hiroshi Kobayashi; hirokoba@hama-med.ac.jp)
}

\begin{abstract}
Bikunin, a Kunitz-type protease inhibitor, is found in blood and urine. It has been established by two laboratories independently that the bikunin knockout female mice display a severe reduction in fertility: the cumulus oophorus has a defect in forming the extracellular hyaluronanrich matrix during expansion. Proteins of the inter-alphatrypsin inhibitor (ITI) family are eliminated in mice in which the bikunin gene has been inactivated, since bikunin is essential for their biosynthesis. Proteins of the ITI family may contribute to the microenvironment in which ovulation takes place. It is not clear, however, whether a single mechanism affects the reproductive function including ovulation. For identifying the full repertoire of the ITI deficiency-related genes, a cDNA microarray hybridization screening was conducted using mRNA from ovaries of wild-type or $b i k^{-1-}$ female mice. A number of genes were identified and their regulation
\end{abstract}

was confirmed by real-time RT-PCR analysis. Our screen identified that $29(0 \cdot 7 \%)$ and 5 genes $(0 \cdot 1 \%)$ of the genes assayed were, respectively, up- and down-regulated twofold or more. The identified genes can be classified into distinct subsets. These include stress-related, apoptosis-related, proteases, signaling molecules, agingrelated, cytokines, hyaluronan metabolism and signaling, reactive oxygen species-related, and retinoid metabolism, which have previously been implicated in enhancing follicle development and/or ovulation. Real-time RTPCR analysis confirmed that these genes were up- and down-regulated two- to tenfold by bikunin knockout. These studies demonstrate that proteins of the ITI family may exert potent regulatory effects on a major physiological reproductive process, ovulation.

Journal of Endocrinology (2004) 183, 29-38

\section{Introduction}

The inter-alpha-trypsin inhibitor (ITI) family members are synthesized and assembled in liver and secreted into the blood at high concentrations $(0.5 \mathrm{mg} / \mathrm{ml}$ ) (Mizon et al. 1996). The members are composed of a common light chain, bikunin, and one or two of the three genetically different heavy chains (HC1, HC2, and HC3) (Salier et al. 1996). Although bikunin was originally identified as a Kunitz-type protease inhibitor essential for inhibition of trypsin and plasmin, there is now increasing evidence to indicate a role for this glycoprotein in the regulation of cell biology (Fries \& Blom 2000). Bikunin may be important in inhibiting the inflammatory response: it has been shown to inhibit the induction of pro-inflammatory cytokines in several types of cells (Nakamura et al. 1997, Futamura et al. 1999, Fries \& Blom 2000, Kobayashi et al. 2002). It also has potent effects on cancer invasion and metastasis. Interestingly, in the case of neoplastic cells, exposure of bikunin or bikunin gene transfection to cancer cells induces suppression of invasion and metastasis (Kobayashi et al. 2002, Suzuki et al. 2003a). Bikunin may modulate different intracellular signaling pathways including MAP (ERK) and phosphoinositide-3 (PI3) kinases (Akt), which lead to the down-regulation of the expression of urokinasetype plasminogen activator (uPA) (Kobayashi et al. 2001, 2003) and its receptor, uPAR (Nakamura et al. 1997).

A role for bikunin in reproductive physiology has recently been recognized (Sato et al. 2001, Zhuo et al. 2001). Mice deficient in bikunin have been generated (Sato et al. 2001, Zhuo et al. 2001). These mice are infertile, with abnormalities in ovulation due to lack of cumulus oocyte complex (COC) expansion (Sato et al. 2001, Zhuo et al. 2001). The heavy chains (HCs) of the ITI family have been shown to bind covalently to hyaluronan (HA) to form the extracellular HA-rich matrices on the COC. Therefore, the formation of the HCs-HA complex (the COC expansion) in mice is abolished by 
targeting the gene of bikunin, which is essential for biosynthesis of ITI (Sato et al. 2001, Zhuo et al. 2001).

Ovarian histology (Sato et al. 2001, Zhuo et al. 2001) revealed that the $b i k^{-1-}$ ovaries appeared normal, because those of 4-week-old females responded normally to gonadotropin treatment, and those of the adults included follicles at all maturation stages, as well as the well defined corpus luteum. However, after a gonadotropin surge in $b i k^{-1-}$ females, the cumulus oophorus matrix fails to form, and the cumulus cells were dispersed in the antral cavity. During cumulus expansion and ovulation, the blood-follicle barrier opens and allows the influx of members of the ITI family from the systemic circulation. Immunohistochemistry revealed that heavy chains of the ITI family colocalized with HA perfectly throughout the matrix network (Zhuo et al. 2001). This finding indicates that the HCs-HA complex is a major component of the cumulus matrix. Therefore, the infertility of $b i k^{-1-}$ females was due to the impaired fertilization of the cumulus oophorus-free oocytes.

Although it is clear that anti-inflammatory homeostasis or many aspects of female reproduction may be regulated by proteins of the ITI family, the precise contribution of the ITI remains to be defined. Presumably, there are select target genes that ITI is capable of regulating in the ovary.

To understand better how proteins of the ITI family regulate different facets of reproductive physiology, we sought to identify ITI-regulated genes in $b i k^{-/-}$mice using microarray technology. There has not been any attempt to systematically define the full repertoire of endogenous ITI-regulated genes. Identification of these genes is required not only for revealing the nature of all signaling pathways used by ITI but also for defining the set of proteins that are induced or repressed by this glycosaminoglycan. In the current study, we started this investigation using a cDNA microarray hybridization analysis of RNA isolated from ovaries from $b i k^{-/-}$and wild-type (WT) female mice. Here, we show that we have identified at least 34 bikunin target genes, and that ITI-selective targets include genes involved in the regulation of signal transduction modifiers, cytokines, apoptosis, ovulation-related, aging-associated, stress-related, and neuroendocrine factors. The encoded proteins are involved in a broad range of cellular functions and signaling pathways.

\section{Materials and Methods}

\section{Animals}

A bik-null mutation, $b i k^{-/-}$, was created in mouse embryonic stem cells, and mice with germline transmission of this mutation were bred, as described previously (Sato et al. 2001). Mice were kept at $25^{\circ} \mathrm{C}$ with a $13-\mathrm{h}$ light, 11-h darkness cycle; a pelleted diet was available ad libitum. Heterozygous mutants were intercrossed to generate $+/+,+/-$, and $-/-$ mice. Pups were genotyped by PCR amplification using tail DNA. To stimulate follicular growth and ovulation (superovulation), 35-dayold females were injected i.p. with 5 IU pregnant mare's serum gonadotropin (PMSG) (Calbiochem, San Diego, CA, USA), and 48 h later with 5 IU human chorionic gonadotropin (hCG) (Calbiochem). Samples were obtained from ovaries from 3 individual mice before gonadotropin injection and $12 \mathrm{~h}$ after hCG injection. Samples from ovaries from 12 individual mice, used as 2 independent experimental samples for each group, were analyzed to determine RNA transcript levels. Animals were used in compliance with the NIH guidelines and with the approval of the animal care review committee of Hamamatsu University.

\section{Tissue processing and preparation of $R N A$}

Fragments of normal ovarian tissue were dissected and homogenized with a rotary homogenizer. Total RNA from samples, as indicated, was prepared using the Trizol reagent (Invitrogen) by standard procedures. RNA integrity was verified by OD 260/OD $280 \mathrm{~nm}$ absorption (Bio-Rad). Total RNA was reverse transcribed as described earlier (Bieser et al. 1998). All other chemicals were analytical grade. The following primer sets were selected based on published data as indicated (some of these data are from Dr Y Yoshikawa, TaKaRa Bio Inc., Otsu, Japan). To verify specificity of each gene amplification, PCR products were isolated and sequenced.

18S ribosomal RNA (18S): forward 5'-AAG TCT TTG GGT TCC GGG-3', reverse 5'-GGA CAT CTA AGG GCA TCA CA-3'; heat_shock_protein_25_kDa_ 2_(cardiovascular) (NM_013868): forward 5'-ACA TCA AGA CCC TCG GGG AT-3', reverse 5'-CTC AGA TTT TTA TCT CCG TCC GGA AG-3'; plasminogen (NM_008877): forward 5'-AGA CAA ACC CAC GGG CAG-3', reverse 5'-GGC TAT TAA AGT ACC GCC ACA GAA-3'; G_protein-coupled_receptor_90 (NM_030726): forward 5'-TCT GGT TTG GAA AAC TTC TTC TGC A-3', reverse 5'-AGT CCC ATA CAG AAT CAT CAA GAG AAC TA-3'; Rad51_ homolog_c_(S._cerevisiae) (NM_053269): forward 5'GCG GGA AGC TGG TGG CTG-3', reverse 5'-AAC TTC TGT CGT CTT CAT TAG GGG TA-3'; protein_kinase_C,_gamma (NM_011102): forward 5'-AAA TTG CAC CTC CTT TCA GAC CA-3', reverse 5'-GAC TAG AGA GGG TAG ATG GGT GTT-3'; calcium_channel,_voltage-dependent,_gamma_subunit_3 (NM_019430): forward 5'-AAG TCT ACA TTT GCG CGC CT-3', reverse 5'-GGG CGT GGT GCG TCT GTT-3'; tumor_necrosis_factor_receptor,_member_ 11b_ (osteoprotegerin) (NM_008764): forward 5'-TGT GAG GAA GGG CGT TAC CT-3', reverse 5'CTC TTC ACA CAG GGT GAC ATC TAT T-3'; tumor_necrosis_factor_receptor_superfamily,_member_17 
(NM_011608): forward 5'-TGA CTT CCT GTC CAC AGG GAA-3', reverse 5'-GAA AAG TGC CAA AGA GAG GAC CA-3'; insulin_receptor-related_receptor (NM_011832): forward 5'-TGT GGG AGA TCA TGA GAG GCT-3', reverse 5'-GGT CCT AGG GTT CCT GCT TT-3'; colony_stimulating_factor_3_(granulocyte) (NM_009971): forward 5'-ACT GTC AGC GCT CTG CCA CCA T-3', reverse 5'-GAA ATA CCC GAT AGA GCC TGC A-3'; klotho_beta (NM_031180): forward 5'-GAA GGC AGC CGA GCG CTT-3', reverse 5'CTT AGG CGG GTG ATG TCC TG-3'; corticotropin_releasing_hormone_receptor (NM_007762): forward 5'-TGG AAA CCC TGC AGC AGT TT-3', reverse 5'-AAT CCT GGT GGC TCA GGA GT-3'; zinc_finger_protein_2,_Y_linked (NM_009571): forward 5'-GTG TGA GTA CTG TGA ATA TAG CAC CAA A-3', reverse 5'-AGC AAT ATC ATA TTT ATA TTC ACT TGA CAA ATT CAT-3'; glutathione_peroxidase_2 (NM_030677): forward 5'-TGC CAT CTA GAT GAG AGC TGC T-3', reverse 5'-CAG ACT GGC TCT GGA CCC TT-3'; retinol_binding_protein_7,_cellular (NM_022020): forward 5'-GCG GAC AGA CAC CAA ACC AT-3', reverse 5'-GTT TGT CAT TCT CCC AGG TAA CCA-3'; synaptotagmin_12 (NM_134164): forward 5'-TGA CCA GCG GAC CAC TAC T-3', reverse 5'-TCC AGG AGG TCT TTG CCT TGT A-3'; myelin-associated_glycoprotein (NM_010758): forward 5'-GCT TGC CTA GCA GAG AAC GCC TAT-3', reverse 5'-TCT CTG GCC ATA CTG GTT CTC A-3'; myelin_oligodendrocyte_glycoprotein (NM_010814): forward 5'-AGG ACA ATT CAG AGT GAT AGG ACC A-3', reverse 5'-AGA AGC AGG TGT AGC CTC CTT-3'; oxidative_stress_induced_protein (U40930): forward 5'-TTA TAG CGA GTT CCC ACC AC-3', reverse 5'-CTC TTT AAT GTA GAT GCG GA-3'; PI-6 (U25844): forward 5'-AGC AGA GTT GGA AGA GC-3', reverse 5'-TCG GGA CTC CTC TGT AG-3'; cathepsin B (X06086): forward 5'-GAA GAA GCT GTG TGG CAC TG-3', reverse 5'-GTT CGG TCA GAA ATG GCT TC-3'.

\section{Microarray probe labeling and hybridization}

Our goal was to determine the gene expression profile in ovaries of gonadotropin-stimulated bikunin knockout (KO) mice. Initially the cDNA microarrays (TaKaRa Mouse IntelliGene II; TaKaRa Bio Inc., Otsu, Japan; http://www.takara-bio.co.jp) were spotted with 4277 sequence-verified clones that are available from GenBank (http://www.ncbi.nlm.nih.gov/Genbank/index.html).

Probes for cDNA microarrays were generated using total RNA from ovaries in a standard reverse transcriptase reaction in which some of the dTTP was replaced with either Cy3-labeled dUTP or Cy5-labeled dUTP (Suzuki et al. 2003b). In some experiments, the sample was labeled with Cy3; in others, it was labeled with Cy5, with essentially identical results. Hybridization of both Cy3and Cy5-labeled probes to the same microarray was carried out in a sealed, humid hybridization cassette for about $14 \mathrm{~h}$ at $65^{\circ} \mathrm{C}$. Microarrays slides were washed and then dried by centrifugation at room temperature. For comparison, commercially available mouse cDNA expression microarrays (Atlas Plastic Mouse $5 \mathrm{~K}$ and Atlas Glass Mouse 3.8I Microarray; Clontech Laboratories, Palo Alto, CA,USA) were also used for hybridization. These microarrays were arrayed with 5002 and 3800 mouse genes respectively. In these microarrays, several housekeeping cDNAs are included as positive controls. A complete list of the cDNAs and controls on the microarray as well as the accession numbers is available at GenBank and Clontech's Atlas website (http://atlas.clontech.com). Twenty-one genes were tested for confirmation by realtime RT-PCR analysis.

\section{Microarray data analysis}

To determine the fluorescent intensities of the two dyes for each spot, the fluorescence signals of $\mathrm{Cy} 3-$ and Cy5-tagged cDNA spots on arrays at $532 \mathrm{~nm}$ (Cy3) and $635 \mathrm{~nm}$ (Cy5) were scanned simultaneously and immediately with an Affymetrix 428 Array Scanner and quantitated using a BioDiscovery ImaGene v. 4·2 (TaKaRa Bio Inc.) (Suzuki et al. 2003b). The background-subtracted median ratio value was calculated for each spot, and replicate spots on each slide were averaged. The fluorescence intensities were normalized by applying a scaling factor so that the median fluorescence ratio of all spots with detectable signals above background on each microarray was $1 \cdot 0$. The spots that displayed a twofold or greater difference in fluorescence intensities between the two dyes were used to generate gene clusters. Poor quality spots were removed if they were very small, irregularly shaped, or with pixels that were not uniformly distributed throughout the spot.

Quantification by SYBR Green-based real-time RT-PCR on LightCycler

In order to validate the relative change in gene expression in ovaries of $b i k^{-1-}$ mice in selected genes identified by the cDNA microarrays, we used a real-time quantitative reverse transcriptase-polymerase chain reaction approach (Castello et al. 2002, Naciff et al. 2002). Ovaries were removed from WT and $b i k^{-1-}$ mice $12 \mathrm{~h}$ after hCG injection ( $n=3 \mathrm{mice} /$ group). Total RNA was extracted from ovaries. A $10 \mu \mathrm{l}$ real-time PCR reaction mix (Roche Diagnostics) was prepared containing the following components: $1 \mu \mathrm{l}$ LightCycler mix, $\mathrm{MgCl}_{2}(4 \mathrm{mM})$, primers $(0 \cdot 4 \mathrm{mM})$ and sample cDNA $(0 \cdot 5 \mathrm{ng} / \mu \mathrm{l})$. Real-time PCR was performed in the Real Time PCR Detection System to continuously monitor the fluorescence of the high affinity, double-stranded, DNA binding dye SYBR Green 
I, using an automated detector combined with special software (Bio-Rad). Absence of genomic DNA contamination in the total RNA samples was confirmed by performing the same RT reactions but without reverse transcriptase, followed by quantitative PCR. All data were normalized to an internal standard (18S ribosomal RNA). Each sample was analyzed in triplicate. Moreover, we confirmed the amplification specificity from each primer pair. At the end of the experiments, RT-PCR products were removed from capillaries and analyzed by gel electrophoresis to confirm the presence and assess the purity of the amplicons of interest. Preliminary experiments were carried out with each primer pair to determine the overall quality and specificity of the primer design. After RTPCR, only the expected products at the correct molecular weight were identified.

\section{Results}

Microarray analysis of ovaries from bik $^{-1-}$ and WT mice

Total RNA was purified from both animal samples, and labeled with either Cy3 or Cy5 fluorescence and then hybridized to the TaKaRa Mouse IntelliGene DNA CHIP. Each slide contained duplicate sets of samples, and the colors of the two cDNA probes were reversed in duplicate assays. Quantitation of the signals produced two kinds of information: the intensity of the signal was proportional to the abundance of the corresponding mRNA, and the degree of redness or greenness indicated the fold induction or repression of mRNA by $b i k^{-/-}$ mice. The average hybridization intensity across all probe sets from the $b i k^{-1-}$ ovary was normalized to that obtained from the WT ovary. Based on the number of genes expressed in WT versus bik ${ }^{-1-}$ samples, as well as on the level of expression of individual genes, the overall gene expression pattern was similar between WT and $b_{i k}{ }^{-1-}$ ovaries (data not shown). For each gene, the intensity ratio of $b i k^{-1-} / \mathrm{WT}$ was calculated. Ratios of 1 indicate equal intensities and therefore no change in gene expression between these ovaries. Ratios below 0.5 indicate down-regulation of gene expression in $b i k^{-/-}$; on the other hand, ratios above $2 \cdot 0$ indicate an up-regulation of gene expression in $b i k^{-1-}$. Using this criterion, we found that 29 and 5 genes were up- and down-regulated respectively in bik $^{-1-}$ (Table 1$)$.

Summary of genes induced or repressed in $\mathrm{bik}^{-1-}$ ovary

Analysis of the results indicated several genes that were altered in $b i k^{-1-}$. Cellular functions of some but not all of the bikunin-related genes identified by our screen are known. In Table 1 , the proteins encoded by these genes are grouped according to their functions. The identified genes can indeed be classified into distinct subsets. These include stress-related, apoptosis-related, proteases, signaling molecules, ion channel, cytokines and growth factors, aging-related, hyaluronan metabolism and signaling, reactive oxygen species-related, retinoid metabolism, and neuroendocrine factors.

From the genes represented in the cDNA microarray used in these studies, many genes previously known to be regulated by gonadotropins were in fact markedly up-regulated in bik ${ }^{-/-}$mice, including apoptosis regulator, stress-induced protein, heat shock proteins (HSPs), cathepsins, and cytokines among others. However, some of the genes responsive to gonadotropin exposure, identified in the present study, had not previously been identified. The genes showing the most robust response (by fold change) to gonadotropin exposure to the $b i k^{-\prime-}$ mice include oxidative stress-induced protein, Cdc42, HSPs, tumor necrosis factor receptor (TNFR), Bcl-w, cathepsins, SPI3, Klotho, Rad51, protein kinase C (PKC), uPA, granulocyte-colony-stimulating factor (G-CSF), corticotropin releasing factor receptor (CRFR), glutathione peroxidase, and retinol binding protein. Therefore, most of the genes are involved in modulation of reproductive biology including ovulation.

\section{Validation of a microarray results using real-time RT-PCR}

To validate the data obtained in the microarray analysis, we have subjected the RNA samples from the cells that were originally used for microarray analysis to real-time quantitative RT-PCR analysis. Fold induction calculated from the microarray data was compared with that obtained using real-time RT-PCR analysis (Table 1 and Fig. 1). The 21 genes were selected for secondary confirmation based on a combination of cDNA probe availability and putative gene function because of interesting properties of the encoded proteins. As shown in Fig. 1, all of the 16 candidate genes were strongly elevated by bikunin knockout, although the level of $18 \mathrm{~S}$ ribosomal mRNA was unchanged. On the other hand, the five candidate genes were repressed by knockout. For many of the genes examined, the actual fold induction by real-time RTPCR was significantly greater than that derived from the microarray analysis (Table 1). Quantitation of the realtime RT-PCR signals revealed that the fold changes observed in the microarray analysis were, in general, underestimates. Thus, even a relatively small difference noted in the microarray analysis may be physiologically significant. This analysis cannot exclude the possibility that stimulation/repression occurs indirectly through activation/inactivation of one or more intermediary molecules.

\section{Discussion}

It has been reported that the mechanism of bikuninmediated inhibition of ovulation is not a direct effect of 
Table 1 Identification of target genes up- and down-regulated in bik ${ }^{-1-}$ mice. The bikunin knockout-related genes can be divided into several large categories. The table lists genes that were found to be up- and down-regulated more than twofold in the bik ${ }^{-1-}$ mice

\begin{tabular}{|c|c|c|c|}
\hline & Fold change & & \\
\hline & Microarray & Real-time RT-PCR & Description \\
\hline $\begin{array}{l}\text { Gene accession } \\
\text { Genes overexpre }\end{array}$ & nin KO mice & & \\
\hline Stress-related & & & \\
\hline U40930 & $5 \cdot 83$ & $7 \cdot 71$ & Oxidative stress induced protein \\
\hline M36829 & $5 \cdot 12$ & & Heat shock protein $84 \mathrm{kDa}$ \\
\hline X53584 & $3 \cdot 83$ & & Heat shock protein $60 \mathrm{kDa}$ \\
\hline M36830 & $2 \cdot 59$ & & Heat shock protein $86 \mathrm{kDa}$ \\
\hline NM_013868 & $2 \cdot 58$ & $2 \cdot 45$ & Heat shock protein 25 kDa 2 (cardiovascular) \\
\hline Apoptosis-relates & & & \\
\hline U59746 & $4 \cdot 25$ & & Bcl-w (bcl-w) mRNA \\
\hline U83628 & $3 \cdot 12$ & & Defender against cell death 1 protein (DAD1) \\
\hline Proteases & & & \\
\hline U25844 & $4 \cdot 07$ & $5 \cdot 92$ & SPI3 serpin b6 \\
\hline M14222 & $4 \cdot 05$ & & Cathepsin B1 \\
\hline X06086 & $4 \cdot 23$ & $4 \cdot 89$ & Cathepsin L \\
\hline NM_013542 & $2 \cdot 69$ & & Granzyme B \\
\hline NM_008877 & $2 \cdot 59$ & $3 \cdot 58$ & Plasminogen \\
\hline NM_008873 & $2 \cdot 13$ & & UPA \\
\hline Signaling molecu & & & \\
\hline U37720 & $5 \cdot 13$ & & CDC42 GTP binding protein \\
\hline NM_030726 & $3 \cdot 44$ & $6 \cdot 13$ & G protein coupled receptor 90 \\
\hline NM_053269 & $3 \cdot 18$ & $6 \cdot 42$ & Rad51 homolog c (S. cerevisiae) \\
\hline NM_011102 & $2 \cdot 93$ & $5 \cdot 17$ & Protein kinase $\mathrm{C}$, gamma \\
\hline Ion channel & & & \\
\hline NM_019430 & $3 \cdot 64$ & $5 \cdot 27$ & Calcium channel, voltage-dependent, gamma subuint 3 \\
\hline Cytokines and g & & & \\
\hline NM_008764 & $4 \cdot 52$ & $6 \cdot 23$ & Tumor necrosis factor receptor $11 \mathrm{~b}$ (osteoprotegerin) \\
\hline NM_011608 & $3 \cdot 67$ & $5 \cdot 11$ & Tumor necrosis factor receptor superfamily, member 17 \\
\hline L20048 & $3 \cdot 51$ & & IL-2 receptor gamma chain \\
\hline NM_011832 & $2 \cdot 81$ & $2 \cdot 39$ & Insulin receptor-related receptor \\
\hline NM_009971 & $2 \cdot 79$ & $3 \cdot 08$ & Colony stimulating factor 3 (granulocyte) \\
\hline D90205 & $2 \cdot 16$ & & IL-5 receptor \\
\hline Aging-related & & & \\
\hline NM_031180 & $3 \cdot 56$ & $4 \cdot 00$ & Klotho beta \\
\hline Hyaluronan met & signaling & & \\
\hline NM_009384 & $2 \cdot 64$ & & Tiam1 \\
\hline Others & & & \\
\hline NM_009571 & $3 \cdot 19$ & $5 \cdot 26$ & Zinc finger protein $2, \mathrm{Y}$ linked \\
\hline NM_007762 & $2 \cdot 58$ & $2 \cdot 66$ & Corticotropin releasing hormone receptor (CRFR) \\
\hline Genes repressed & KO mice & & \\
\hline Reactive oxygen & & & \\
\hline NM_030677 & $0 \cdot 14$ & $0 \cdot 11$ & Glutathione peroxidase 2 \\
\hline Retinoid metabo & & & \\
\hline NM_022020 & $0 \cdot 22$ & $0 \cdot 15$ & Retinol binding protein 7 , cellular \\
\hline Neuroendocrine & & & \\
\hline NM_134164 & $0 \cdot 27$ & $0 \cdot 13$ & Synaptotagmin 12 \\
\hline NM_010758 & $0 \cdot 31$ & $0 \cdot 24$ & Myelin-associated glycoprotein \\
\hline NM_010814 & $0 \cdot 37$ & $0 \cdot 19$ & Myelin oligodendrocyte glycoprotein \\
\hline
\end{tabular}

${ }^{*}$ The numbers indicate the fold induction of each gene as determined by the microarray analysis software or real-time quantitative RT-PCR analysis. 


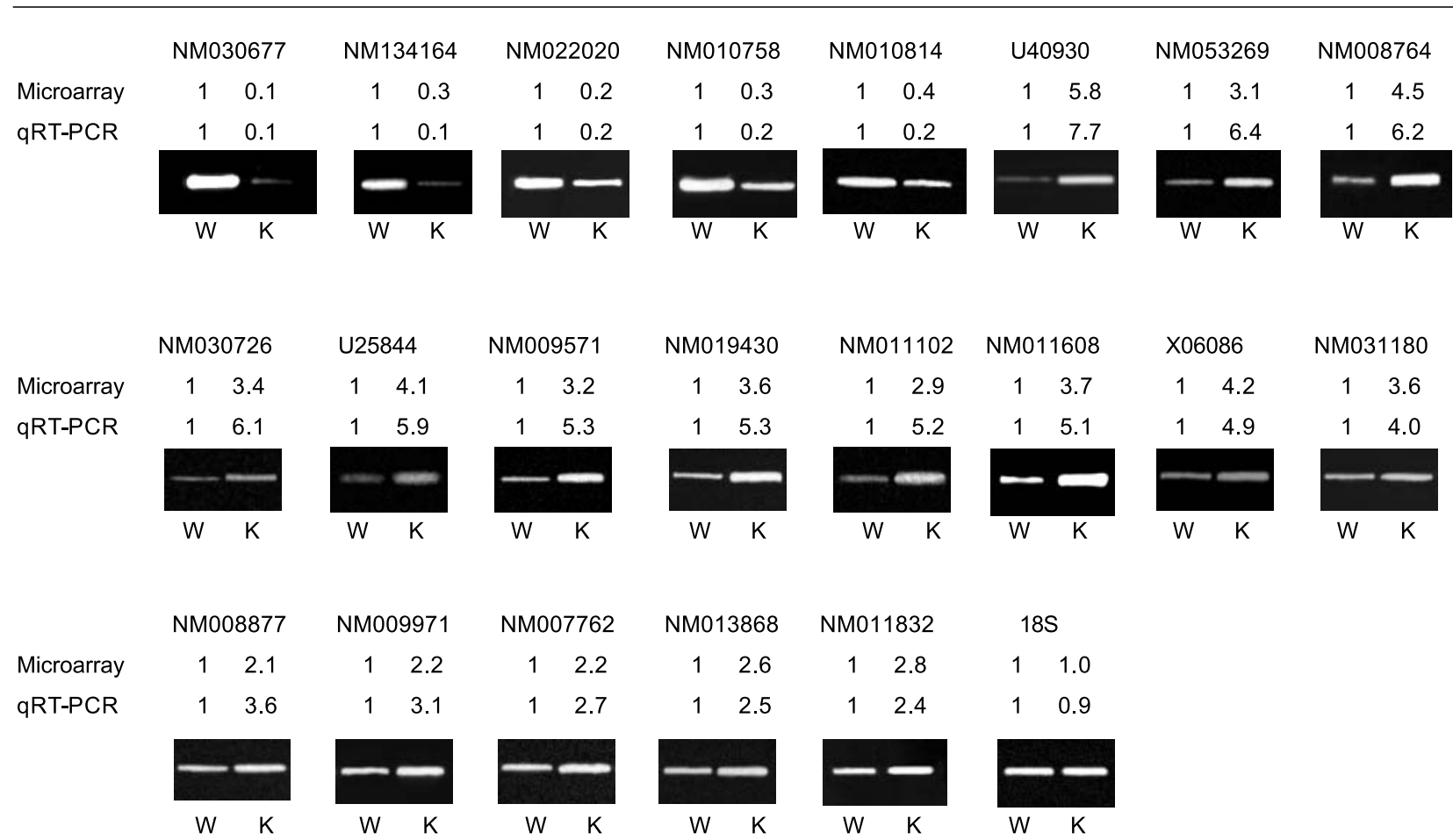

Figure 1 Confirmation of microarray-determined gene-expression changes by quantitative RT-PCR (qRT-PCR). Representative images of RT-PCR products for the indicated genes, separated in agarose gels and visualized, after ethidium bromide staining, with UV light. Relative values of expression of the indicated genes quantified by real-time RT-PCR are shown in Table 1, indicating the comparison of the fold change determined by microarray or by real-time RT-PCR analysis for the indicated genes. W, wild-type; K, knockout.

genetic down-expression of bikunin protein but most likely occurs via suppression of ITI production (leading to lack of HCs-HA complex on cumulus cells), since bikunin is considered to play an important role in ITI production (Zhuo et al. 2001). In the present study we investigated whether proteins of the ITI family have a direct effect on reproductive biology including ovulation. To understand the molecular mechanism underlying the ovulation defect caused by genetic bikunin deletion, we set out to identify gene expression pathways that might be disrupted in our ITI-deficient ovaries. To address the mechanisms that explain these functions, we sought to identify bikunin knockout-related genes using microarray analysis.

Here, we show that down-expression of ITI by targeted disruption of the bikunin gene altered the expression of several genes. Only $29(0.7 \%)$ and 5 genes $(0 \cdot 1 \%)$ of the genes assayed were up- and down-regulated respectively by twofold or more. All of the altered gene expressions were confirmed by real-time quantitative RT-PCR analysis. A number of genes known to function in folliculogenesis and known to be important for female fertility were constitutively up-regulated (2- to 10-fold) in the ITI-deficient ovaries (Table 1). Some of these genes are considered to be associated with the ovulation-related group.

Most notably, the expressions of stress-related, apoptosisrelated, proteases, signaling molecules, cytokines, hyaluronan metabolism, and aging-related pathways were constitutively up-regulated in the ITI knockout ovaries. Consistent with the role of this specific set of genes, up-regulating their expression correlates with the improper regulation of folliculogenesis and the ovulatory system seen in the ITI-deficient ovaries and may explain the infertility observed in these mice.

\section{Heat shock protein}

Heat shock response of mouse oocytes is maximal during the growth period, significantly declines with acquisition of full oocyte size and antrum formation within the follicle, and is finally shut off with oocyte/follicle terminal differentiation (Curci et al. 1991). In addition, the findings that the synthesis of HSPs coincides with the loss of luteal function and that blocking HSP synthesis reverses inhibition of hormone-dependent steroidogenesis strongly suggest a role for HSPs as physiological mediators of luteal regression (Khanna et al. 1995). In view of known functions of the HSPs, it is likely that these proteins are synthesized in response to adverse environmental conditions (Hahnel et al. 1986). Further, HSPs are believed to prevent damage to protein structures under various conditions of environmental stress (Tanake et al. 1995, Hashizume et al. 1997, Neuer et al. 1998). We speculate that the ITI KO ovary may constitutively produce HSPs to 
conquer adverse environmental conditions. In addition, tumor necrosis factor (TNF)- $\alpha$ is a cytotoxic cytokine that is produced in progressive amounts during the secretory phase. It has been reported that bikunin has an ability to inhibit expression of several cytokines including TNF- $\alpha$ by inflammatory cells or neoplastic cells (Kobayashi et al. 2001). Here, we show that TNF- $\alpha$ receptor (osteoprotegerin; see below) is constitutively overexpressed in $b i k^{-/-}$ mouse ovary. The function of the HSPs may be to protect cells against the cytotoxic damage of TNF- $\alpha$.

\section{Osteoprotegerin}

Osteoprotegerin (OPG) is a novel member of the TNF receptor superfamily (Simonet et al. 1997). Interestingly, OPG mRNA was localized in granulosa cells and was detected in the ovary during and after natural ovulation. Its expression was up-regulated at the end of ovulation and progressively down-regulated after $48 \mathrm{~h}$ postovulation. However, we show that OPG is constitutively overexpressed in $b i k^{-1-}$ mouse ovary.

\section{$B c l-w$}

The majority of ovarian follicles undergo atresia mediated by apoptosis (Leo et al. 1999). One key group of intracellular factors regulating apoptosis is the $\mathrm{Bcl}-2$ family of proteins (Adams \& Cory 1998). A delicate balance between anti- (Bcl-2 and Bcl-xL and Bcl-w) and pro(Bax and BAD) apoptotic Bcl-2 family members exists in each cell, and the relative concentrations of these two groups of proteins determine whether the cell survives or undergoes apoptosis. We speculate that $\mathrm{Bcl}-\mathrm{w}$ may be constitutively overexpressed in bik ${ }^{-1-}$ mouse ovary to overcome adverse microenvironmental conditions as a defence mechanism.

\section{Cdc42}

The Rho family of low molecular weight GTP-binding proteins is important for cadherin-mediated adhesion. Several lines of evidence suggest that members of the Rho family are required for the establishment and maintenance of cadherin-based adherence junctions (Noren et al. 2001). Overexpression of constitutively active Rac1 or Cdc42 increases E-cadherin localization and actin accumulation at cell-cell junctions (Noren et al. 2001). It has been established that the specific decrease of $\alpha$-catenin in granulosa cells and the reduction of both $\alpha$-catenin and E-cadherin in theca cells of ovulatory follicles might reflect some of the molecular changes in cell-cell adhesion associated with COC expansion, ovulation and luteinization (Sundfeldt et al. 2000). These data allow us to speculate that consti- tutive overexpression of $\mathrm{Cdc} 42$ may prevent COC expansion possibly via up-regulation of cadherins at the preovulatory follicle.

\section{Serine protease inhibitor 6}

The intracellular serpin, proteinase inhibitor 6 (PI-6), is present in monocytes and granulocytes. PI-6 specifically binds and neutralizes cathepsin that leaks into the cytoplasm of monocytes or granulocytes during biosynthesis or phagocytosis. Control of intracellular cathepsin G may be particularly important, because it has recently been shown to activate the proapoptotic proteinase, caspase-7 (Scott et al. 1999). Overexpression of PI-6 may prevent activation of caspase-7 during folliculogenesis.

\section{Cathepsins}

The expression of cathepsin L (a member of the papain family) and ADAMTS-1 (A Disintegrin And Metalloproteinase with Thrombospondin-like motifs) are selectively induced in granulosa cells of preovulatory follicles by the luteinizing hormone (LH) surge (Robker et al. 2000). Maximal levels of these proteases are observed at 12-16 h after an LH surge, the time of ovulation. These novel observations indicate that these two proteases regulate some key step(s) controlling ovulation. The constitutive overexpression of cathepsins may destroy the finely tuned mechanism of ovulation.

\section{Cytokines}

There are interactions between the immune and reproductive systems. The ovary contains indigenous macrophages, as well as other classes of leukocytes. Cytokines secreted by these cells have been shown to have the ability to regulate ovarian steroidogenesis. Further, cytokines have a role in reproductive functions such as ovulation and pregnancy. It has been reported that the estrous cycles in interleukin (IL)-2 gamma-chain KO mice were irregular compared with wild-type mice (Miyazaki et al. 2002). Macrophage/leukocyte-derived IL-5 significantly increased the 11 beta-hydroxysteroid dehydrogenase activity in granulosa cells (Evagelatou et al. 1997). Thus, leukocytes interact with the ovarian cells through cytokine secretion and/or cell-cell contact to regulate steroidogenesis in human granulosa cells.

\section{Hyaluronan metabolism and signaling}

Tiam1 (T lymphoma invasion and metastasis 1) has been identified as an oncogene because of its ability to activate Rho-like GTPases during malignant transformation (van Leeuwen et al. 1995). The linkage between CD44 and Tiam1 is required for hyaluronan-stimulated Rac1 signaling and cytoskeleton-mediated signaling (Bourguignon 
et al. 2000). In addition, Cdc37, a cell cycle regulatory protein, is identified and recognized as a novel group of hyaluronan-binding proteins (Huang et al. 2000).

\section{Klotho}

A unique short lifespan mouse strain in which a single gene mutation caused multiple aging-related disorders has recently been developed and the gene responsible has been identified as klotho (Nabeshima 2002). This gene is associated with the suppression of several aging phenotypes (Nagai et al. 2003). In addition, oxidative stress has a crucial role in the aging-associated cognition impairment in klotho mutant mice, demonstrating that Klotho protein may be involved in the regulation of antioxidative defence (Nagai et al. 2003). These results suggest that induction of the klotho gene may have protective effects in ovarian damage in $b^{-1 k^{-1-}}$ mouse.

\section{Granulocyte-colony stimulating factor}

Normal ovarian surface epithelial cells secrete G-CSF that is known to have regulatory effects on follicular growth and differentiation, ovulation, and the distribution of intraovarian cells of the immune system (Ziltener et al. 1993). Ovulation has several similarities with inflammation and is closely connected to the activity of leukocytes and inflammatory cytokines. G-CSF is produced in the human follicle shortly before the ovulatory phase and may play an important role in the mechanism of ovulation (Makinoda et al. 1995, 1996, Yanagi et al. 2002). It is likely that the constitutive overexpression of G-CSF may destroy a finely tuned mechanism of ovulation.

\section{Protein kinase $C$}

Recent reports indicate that PKC may play an important role in the process of gonadotropin-induced ovulation in the ovary (Shimamoto et al. 1993). Estradiol production in granulosa cells from large preovulatory follicles may come under the stimulatory control of regulators of PKC as in follicles near ovulation (Hylka \& diZerega 1990). Here we show that the PKC level is constitutively overexpressed in the $b i k^{-1-}$ mouse ovary.

\section{Glutathione peroxidase}

The fundamental defence of the organism against reactive oxygen species (ROS) includes scavenger enzymes (superoxide dismutase, catalase, glutathione peroxidase) and lipid- and water-soluble antioxidant compounds (ascorbic acid, glutathione, albumin, transferrin, etc.) (Roth 1997). Here we show that glutathione peroxidase levels are constitutively repressed in the $b i k^{-1-}$ mouse ovary. The function of glutathione peroxidase may be to protect cells against the cytotoxic damage of ROS, suggesting that the ITI $\mathrm{KO}$ ovary may fail to conquer adverse environmental conditions.

\section{Retinoid-binding protein}

Retinoid-binding proteins and nuclear receptors are expressed in the reproductive tissues of different species and their expression is hormonally regulated (Schweigert \& Siegling 2001). The presence of specific proteins involved in retinoid metabolism was dependent on events associated with ovulation, the migration of the oocyte through the oviduct and the possible implantation of the blastocyst into the uterus (Schweigert \& Siegling 2001). Here we show that the retinoid-binding protein level is constitutively repressed in the $b i k^{-1-}$ mouse ovary.

It has been reported (Sato et al. 2001, Zhuo et al. 2001) that $b i k^{-1-}$ mice are infertile, with abnormalities in ovulation due to lack of COC expansion. Based on the present analysis, we hypothesize that $b i k^{-/-}$mice fail to ovulate possibly through the constitutive up-regulation of HSP, Bcl-w, IL-2 receptor gamma-chain, IL-5 receptor, OPG, Cdc42, protease inhibitor-6 (PI-6), cathepsins, Klotho, G-CSF, PKC, as well as the down-regulation of glutathione peroxidase, retinoid-binding protein, and myelin-associated glycoprotein. Therefore, these results allow us to speculate that proteins of the ITI family in serum or follicular fluid can promote ovulation possibly via improvement of adverse microenvironmental conditions. The molecular basis for this type of specificity may be due to some combination of ITI-dependent interactions with other cofactors or pathways. There may also be unique cell cofactors that play a role in dictating ITI specificity. ITI is a protease inhibitor as well as an extracellular matrix (ECM) stabilizer rather than a transcription factor. Thus, the changes in gene expression are probably due to some form of altered signaling. ITI may positively regulate possible cross-talk between reproductive biology and certain signaling actions. It is not known, however, how many of these genes identified in this study contain functional response elements. It is possible that proteins of the ITI family may regulate these genes via a transactivation/transrepression mechanism that involves activation/competition for limiting amounts of signal molecules.

In conclusion, we have delineated for the first time the biochemical mechanism by which proteins of the ITI family may regulate ovulation by modulating the expression of specific genes. The results presented here should alert us to the fact that proteins of the ITI family will have additional global effects on reproductive biology by modulating the expression of a large number of cellular genes, although it is clear that many aspects of ovulation, especially COC expansion during a preovulatory period, are indeed regulated by HCs of ITI. Clearly, the genomic response to ITI signaling is complex. Future studies 
focused on the regulation and functional significance of the target genes reported here should increase our knowledge of the biological activity of proteins of the ITI family, including bikunin.

\section{Acknowledgements}

We are thankful to Drs $\mathrm{H}$ Morishita, and Y Kato (Research Center, Mochida Pharmaceutical Co., Gotemba, Shizuoka, Japan), Drs Y Tanaka and T Kondo (Chugai Pharmaceutical Co. Ltd, Tokyo, Japan), and Drs S Miyauchi and M Ikeda (Seikagaku Kogyo Co. Ltd, Tokyo, Japan) for their continuous and generous support of our work.

\section{Funding}

This work was supported by a grant-in-aid for Scientific Research from the Ministry of Education, Science and Culture of Japan (to H K and Y T) and by a grant from the Yamanouchi Foundation for Research on Metabolic Disorders.

\section{References}

Adams JM \& Cory S 1998 The Bcl-2 protein family: arbiters of cell survival. Science 281 1322-1326.

Bieser B, Stojkovic M, Wolf E, Meyer H \& Einspanier R 1998 Growth factors and components for extracellular proteolysis are differentially expressed during in vitro maturation of bovine cumulus-oocyte complexes. Biology of Reproduction 59 801-806.

Bourguignon LY, Zhu H, Shao L \& Chen YW 2000 CD44 interaction with tiam1 promotes Rac1 signaling and hyaluronic acid-mediated breast tumor cell migration. Journal of Biological Chemistry 275 1829-1838.

Castello R, Estelles A, Vazquez C, Falco C, Espana F, Almenar SM, Fuster C \& Aznar J 2002 Quantitative real-time reverse transcription-PCR assay for urokinase plasminogen activator, plasminogen activator inhibitor type 1, and tissue metalloproteinase inhibitor type 1 gene expressions in primary breast cancer. Clinical Chemistry 48 1288-1295.

Curci A, Bevilacqua A, Fiorenza MT \& Mangia F 1991 Developmental regulation of heat-shock response in mouse oogenesis: identification of differentially responsive oocyte classes during Graafian follicle development. Developmental Biology 144 362-368.

Evagelatou M, Peterson SL \& Cooke BA 1997 Leukocytes modulate 11 beta-hydroxysteroid dehydrogenase (11 beta-HSD) activity in human granulosa-lutein cell cultures. Molecular and Cellular Endocrinology 133 81-88.

Fries E \& Blom AM 2000 Bikunin - not just a plasma proteinase inhibitor. International Journal of Biochemistry and Cell Biology 32 125-137.

Futamura Y, Kajikawa S, Kaga N \& Shibutani Y 1999 Protection against preterm delivery in mice by urinary trypsin inhibitor. Obstetrics and Gynecology 93 100-108.

Hahnel AC, Gifford DJ, Heikkila JJ \& Schultz GA 1986 Expression of the major heat shock protein (hsp 70) family during early mouse embryo development. Teratogenesis, Carcinogenesis and Mutagenesis 6 493-510.
Hashizume H, Tokura Y, Takigawa M \& Paus R 1997 Hair cycle-dependent expression of heat shock proteins in hair follicle epithelium. International Journal of Dermatology 36 587-592.

Huang L, Grammatikakis N, Yoneda M, Banerjee SD \& Toole BP. 2000 Molecular characterization of a novel intracellular hyaluronan-binding protein. Journal of Biological Chemistry 275 29829-29839.

Hylka VW \& diZerega GS 1990 Granulosa cells from pig follicles of different sizes demonstrate maturational differences in their steroidogenic responses to FSH, calcium ionophore A23187, and phorbol diester. Journal of Reproduction and Fertility 89 181-191.

Khanna A, Aten RF \& Behrman HR 1995 Heat shock protein-70 induction mediates luteal regression in the rat. Molecular Endocrinology 9 1431-1440.

Kobayashi H, Suzuki M, Tanaka Y, Hirashima Y \& Terao T 2001 Suppression of urokinase expression and invasiveness by urinary trypsin inhibitor is mediated through inhibition of protein kinase Cand MEK/ERK/c-Jun-dependent signaling pathways. Journal of Biological Chemistry 276 2015-2022.

Kobayashi H, Suzuki M, Kanayama N, Nishida T, Takigawa M \& Terao T 2002 Suppression of urokinase receptor expression by bikunin is associated with inhibition of upstream targets of extracellular signal-regulated kinase-dependent cascade. European Journal of Biochemistry 269 3945-3957.

Kobayashi H, Suzuki M, Tanaka Y, Kanayama N \& Terao T 2003 A Kunitz-type protease inhibitor, bikunin, inhibits ovarian cancer cell invasion by blocking the calcium-dependent transforming growth factor-beta 1 signaling cascade. Journal of Biological Chemistry 278 7790-7799.

van Leeuwen FN, van der Kammen RA, Habets GG \& Collard JG 1995 Oncogenic activity of Tiam1 and Rac1 in NIH3T3 cells. Oncogene 11 2215-2221.

Leo CP, Hsu SY, Chun SY, Bae HW \& Hsueh AJ 1999 Characterization of the antiapoptotic Bcl-2 family member myeloid cell leukemia-1 (Mcl-1) and the stimulation of its message by gonadotropins in the rat ovary. Endocrinology 140 5469-5477.

Makinoda S, Mikuni M, Furuta I, Okuyama K, Sagawa T \& Fujimoto S 1995 Serum concentration of endogenous G-CSF in women during the menstrual cycle and pregnancy. European Journal of Clinical Investigation 25 877-879.

Makinoda S, Mikuni M, Sogame M, Kobamatsu Y, Furuta I, Yamada H, Yamamoto R \& Fujimoto S 1996 Erythropoietin, granulocyte-colony stimulating factor, interleukin-1 beta and interleukin-6 during the normal menstrual cycle. International Journal Gynaecology Obstetrics 55 265-271.

Miyazaki S, Tanebe K, Sakai M, Michimata T, Tsuda H, Fujimura M, Nakamura M, Kiso Y \& Saito S 2002 Interleukin 2 receptor gamma chain (gamma(c)) knockout mice show less regularity in estrous cycle but achieve normal pregnancy without fetal compromise. American Journal of Reproductive Immunology 47 222-230.

Mizon C, Balduyck M, Albani D, Michalski C, Burnouf T \& Mizon J 1996 Development of an enzyme-linked immunosorbent assay for human plasma inter-alpha-trypsin inhibitor (ITI) using specific antibodies against each of the $\mathrm{H} 1$ and $\mathrm{H} 2$ heavy chains. Journal of Immunological Methods 190 61-70.

Nabeshima Y 2002 Klotho: a fundamental regulator of aging. Ageing Research Reviews 1 627-638.

Naciff JM, Jump ML, Torontali SM, Carr GJ, Tiesman JP, Overmann GJ \& Daston GP 2002 Gene expression profile induced by $17 \alpha$-ethynyl estradiol, bisphenol a, and genistein in the developing female reproductive system of the rat. Toxicology Science $\mathbf{6 8}$ 184-199.

Nagai T, Yamada K, Kim HC, Kim YS, Noda Y, Imura A, Nabeshima Y \& Nabeshima T 2003 Cognition impairment in the genetic model of aging klotho gene mutant mice: a role of oxidative stress. FASEB 17 50-52. 
Nakamura H, Abe S, Shibata Y, Sata M, Kato S, Saito H, Hino T, Takahashi H \& Tomoike H 1997 Inhibition of neutrophil elastase-induced interleukin- 8 gene expression by urinary trypsin inhibitor in human bronchial epithelial cells. International Archives Allergy and Immunology 112 157-162.

Neuer A, Mele C, Liu HC, Rosenwaks Z \& Witkin SS 1998 Monoclonal antibodies to mammalian heat shock proteins impair mouse embryo development in vitro. Human Reproduction 13 987-990.

Noren NK, Niessen CM, Gumbiner BM \& Burridge K 2001 Cadherin engagement regulates Rho family GTPases. Journal of Biological Chemistry 276 33305-33308.

Robker RL, Russell DL, Yoshioka S, Sharma SC, Lydon JP, O’Malley BW, Espey LL \& Richards JS 2000 Ovulation: a multi-gene, multi-step process. Steroids 65 559-570.

Roth E 1997 Oxygen free radicals and their clinical implications. Acta Chirurgica Hungarica 36 302-305.

Salier JP, Rouet P, Raguenez G \& Daveau M 1996 The inter-alpha-inhibitor family: from structure to regulation. Biochemical Journal 315 1-9.

Sato H, Kajikawa S, Kuroda S, Horisawa Y, Nakamura N, Kaga N, Kakinuma C, Kato K, Morishita H, Niwa H \& Miyazaki J 2001 Impaired fertility in female mice lacking urinary trypsin inhibitor. Biochemical and Biophysical Research Communications 281 1154-1160.

Schweigert FJ \& Siegling C 2001 Immunolocalization of retinol-binding protein, cellular retinoic acid-binding protein I and retinoid $\mathrm{X}$ receptor beta in the porcine reproductive tract during the oestrous cycle. Reproduction, Fertility, and Development $\mathbf{1 3}$ 421-426.

Scott FL, Hirst CE, Sun J, Bird CH, Bottomley SP \& Bird PI 1999 The intracellular serpin proteinase inhibitor 6 is expressed in monocytes and granulocytes and is a potent inhibitor of the azurophilic granule protease, cathepsin G. Blood 93 2089-2097.

Shimamoto T, Yamoto M \& Nakano R 1993 Possible involvement of protein kinase $\mathrm{C}$ in gonadotropin-induced ovulation in the rat ovary. Endocrinology 133 2127-2132.

Simonet WS, Lacey DL, Dunstan CR, Kelley M, Chang MS, Luthy R, Nguyen HQ, Wooden S, Bennett L, Boone T, Shimamoto G, DeRose M, Elliott R, Colombero A, Tan HL, Trail G, Sullivan J,
Davy E, Bucay N, Renshaw-Gegg L, Hughes TM, Hill D, Pattison W, Campbell P \& Boyle WJ 1997 Osteoprotegerin: a novel secreted protein involved in the regulation of bone density. Cell 89 309-319.

Sundfeldt K, Piontkewitz Y, Billig H \& Hedin L 2000

E-cadherin-catenin complex in the rat ovary: cell-specific expression during folliculogenesis and luteal formation. Journal of Reproduction and Fertility 118 375-385.

Suzuki M, Kobayashi H, Tanaka Y, Hirashima Y, Kanayama N, Takei Y, Saga Y, Suzuki M, Itoh H \& Terao T 2003a Suppression of invasion and peritoneal carcinomatosis of ovarian cancer cell line by overexpression of bikunin. International Journal of Cancer 104 289-302.

Suzuki M, Kobayashi H, Tanaka Y, Hirashima Y, Kanayama N, Takei Y, Saga Y, Suzuki M, Itoh H \& Terao T 2003 b Bikunin target genes in ovarian cancer cells identified by microarray analysis. Journal of Biological Chemistry 278 14640-14646.

Tanaka Y, Kobayashi K, Kita M, Kinoshita S \& Imanishi J 1995 Messenger RNA expression of heat shock proteins (HSPs) during ocular development. Current Eye Research 14 1125-1133.

Yanagi K, Makinoda S, Fujii R, Miyazaki S, Fujita S, Tomizawa H, Yoshida K, Iura T, Takegami T \& Nojima T 2002 Cyclic changes of granulocyte colony-stimulating factor (G-CSF) mRNA in the human follicle during the normal menstrual cycle and immunolocalization of G-CSF protein. Human Reproduction 17 3046-3052.

Zhuo L, Yoneda M, Zhao M, Yingsung W, Yoshida N, Kitagawa Y, Kawamura K, Suzuki T \& Kimata K 2001 Defect in SHAP-hyaluronan complex causes severe female infertility. A study by inactivation of the bikunin gene in mice. Journal of Biological Chemistry 276 7693-7696.

Ziltener HJ, Maines-Bandiera S, Schrader JW \& Auersperg N 1993 Secretion of bioactive interleukin-1, interleukin-6, and colony-stimulating factors by human ovarian surface epithelium. Biology of Reproduction 49 635-641.

Received 26 April 2004

Accepted 29 June 2004 